\title{
Exosomal miR-375 promotes the activity of osteoblasts in prostate cancer
}

\author{
Suliang $\mathrm{Li}^{1^{*}}$, Yun $\mathrm{Ye}^{1}$, Shengyu Wang ${ }^{1}$, Jianjun $\mathrm{Wang}^{2}$ \\ ${ }^{1}$ Department of Clinical Laboratory, The First Affiliated Hospital of Xi'an Medical University, Xi'an 710077, Shaanxi, China; \\ ${ }^{2}$ Intensive Care Unit, The First Affiliated Hospital of Xi'an Medical University, Xi'an 710077, Shaanxi, China.
}

\begin{abstract}
Studies have shown that exosomes influence tumour metastasis, diagnosis, and treatment. It has been found that exosomal miRNAs are closely linked to the metastatic microenvironment. However, the regulatory role of exosomes from prostate cancer ( $\mathrm{PCa}$ ) cells in bone metastasis remains poorly understood. Here, exosomes were isolated and purified by ultracentrifugation, total RNA from cells and total miRNA from exosomes were isolated, and the level of miR-375 was analyzed by RT-PCR. Exosome libraries from LNCaP cells and RWPE-1 cells were sequenced and filtered with an Illumina HiSeq ${ }^{\mathrm{TM}} 2500$ system. The activity of alkaline phosphatase, the extent of extracellular matrix mineralization, and the expression of osteoblast activity-related marker genes were measured to evaluate osteoblast activity. Morphological observation, particle size analysis, and molecular phenotyping confirmed that the isolated extracts contained exosomes. Differential expression analysis confirmed the high expression of miR-375 in LNCaP cell-derived exosomes. This study suggest that exosomes could enter osteoblasts and increase their miR-375 level. In addition, exosomal miR-375 could significantly promote the activity of osteoblasts. This study lays the foundation for further investigations on the function of exosomal miR-375 in the activation and differentiation of osteoblasts and the mechanism of bone metastasis in PCa.
\end{abstract}

Keywords: prostate cancer, exosome, miR-375, osteoblasts.

\section{INTRODUCTION}

Prostate cancer $(\mathrm{PCa})$ is a common male urinary system malignant tumour, with the highest incidence among European and American populations ${ }^{[1-2]}$. Bone is the most common metastatic target for PCa, and $80 \%$ to $90 \%$ of advanced PCa cases show different degrees of bone metastasis ${ }^{[3]}$. Approximately $50 \%$ of PCa patients with bone metastases die within 2 to 3

\footnotetext{
*Correspondence to: Suliang Li, Department of Clinical Laboratory, The First Affiliated Hospital of Xi'an Medical University, Xi'an 710077, Shaanxi, China. Tel: +86-29-84277552,Fax: +86-29-84277393;E-mail: lisuliang01@163.com.

Conflict of interests: The authors indicate no potential conflicts of interest related to this work.
}

years after diagnosis. This disease not only seriously affects the quality of life of patients, but also brings a heavy financial burden to the society and family ${ }^{[4-5]}$. Bone metastasis is a continuous, multi-step, and multifactorial cascade reaction, involving the transformation of tumour cells and their interaction with the microenvironment. Thus, the bone metastatic process is complicated, and the mechanism remains unclear.

Exosomes are small vesicles with a diameter of approximately 30 to $150 \mathrm{~nm}$, and almost all cells can secrete exosomes under normal and pathological conditions ${ }^{[6]}$. Early studies suggested that exosomes perform protein transport functions, target specific receptor cells, and trigger downstream signalling events ${ }^{[7]}$. Studies prior to 2007 showed that exosomes also transport nucleic acids and are involved in in- 
tercellular communication ${ }^{[8]}$. In recent years, a large number of studies have confirmed that tumourrelated exosomes play significant roles in tumour metastasis, tumour diagnosis and monitoring, and tumour therapy ${ }^{[9-10]}$. miRNA is a type of non-coding, small RNA that binds to the 3 UTR of targeted mRNAs to regulate and alter the expression of target genes. Previous studies have shown that the expression of mRNA in receptor cells was inhibited by exosomemediated miRNA ${ }^{[11-12]}$. Exosomal miRNA secreted by cancer cells further impacts the status of immune cells and metastatic site cells, which are closely related to systemic immunity and the formation of a metastatic microenvironment ${ }^{[13]}$. However, the regulatory role of exosomes from PCa cells in bone metastasis remains poorly understood.

\section{MATERIALS AND METHODS}

\section{Cell culture}

LNCaP, RWPE-1 and hFOB1.19 cells from ATCC (Manassas, VA, USA) were obtained and hFOB1.19 cells were cultured in DMEM/F12 (HyClone, Logan, UT, USA) and LNCaP and RWPE-1 cells in RPMI-1640 (Gibco BRL Co. Ltd., USA); all culture media were supplemented with $10 \%$ FBS (Gibco), $100 \mathrm{U} / \mathrm{mL}$ of penicillin and $100 \mathrm{mg} / \mathrm{mL}$ of streptomycin (HyClone).

\section{Isolation of exosomes}

Exosomes from cells were isolated according to previously described methods ${ }^{[14]}$. Cells were cultured in FBS-supplemented culture media that were depleted of exosomes. The supernatant was collected after $48 \mathrm{~h}$ of cell culture and then centrifuged $(500 \times g ; 10 \mathrm{~min})$, was filtered $(0.22 \mu \mathrm{m}$, micropores) to remove dead cells and large debris. Subsequently, we collected the exosomes, washed them with PBS, ultracentrifuged them at $100,000 \times \mathrm{g}$ for $90 \mathrm{~min}$ at $4^{\circ} \mathrm{C}$ and resuspended the exosomes in PBS. The NanoSight Tracking Analysis LM20 System (NanoSight Ltd.) was used to examine the size distribution of the exosomes, and the transmission electron microscope (TEM-1400plus) was used to perform microscopic imaging.

\section{Co-culture and transfection experiments}

According to the manufacturer's instructions, transwell chambers with 0.4-mm-pore filters were used (Sigma Aldrich, St. Louis, USA). The osteoblasts were inoculated into the lower chambers and LNCaP or RWPE-1 cells into the top chambers and cultured for $72 \mathrm{~h}$. A lentiviral vector system encoding cytomegalovirus (CMV)-driven RFP-tagged CD63
(CMV-RFP-CD63) was used to label the exosomes (Shanghai SunBio Biomedical Technology Co., Ltd). A pre-experiment was conducted the day before the co-culture experiment according to the manufacturer's instructions, and a multiplicity of infection (MOI) was determined at 100 .

\section{RT-PCR}

The total RNA from cells and total miRNA from exosomes were isolated by using TRIzol (Life Technologies) reagent and the mirVana miRNA Isolation Kit (Ambion). Total RNA was treated with TURBO DNase (Ambion) after purification and a highcapacity cDNA reverse transcription kit (Takara) was used to reverse transcribe RNA into first-strand cDNA. Primers against mRNAs were purchased from Shanghai Sangon Biotech Co., Ltd. The ABI Prism 7500 Sequence Detection System (Applied Biosystems) was used to perform real-time $\mathrm{PCR}^{[14]}$.

\section{Western blotting}

The previously described protocols to harvest cells and extract proteins were followed ${ }^{[15]}$. The protein assay kit (Bio-Rad, Hercules, CA, USA) was used to determine protein concentrations, and SDS polyacrylamide gel electrophoresis was used to separate the samples according to molecular weight. The blotting membrane was incubated with rabbit monoclonal ant-GM130 (1:1,000,ab52649, Abcam,USA), rabbit monoclonal anti-CD63 (1:1,000, ab134045, Abcam,USA), rabbit monoclonal anti-Alix (1:1,000, ab186429, Abcam, USA), and rabbit monoclonal anti-Hsp70 (1:1,000, ab181606, Abcam, USA) antibodies, and horseradish peroxidase (HRP)-coupled goat anti-rabbit IgG H\&L (1:5,000, ab6721, Abcam, USA) as secondary antibodies. Primary antibody dilutions were incubated with the membranes overnight at $4{ }^{\circ} \mathrm{C}$.

\section{Statistical analysis}

Two-tailed Mann-Whitney U-tests were used to analyse non-parametric data. Analysis of variance (ANOVA) was used to analyze parametric data. The $2^{-\Delta \Delta \mathrm{CT}}$ method was used to calculate the relative expression of miRNAs. SPSS 18.0 software(SPSS Inc.) was used to perform statistical analyses, with adjusted $P \leqslant 0.05$ for statistical significance.

\section{RESULTS}

\section{The identification of exosomes in cell cul- ture medium}

Exosomes can be observed as round, oval or cup- 
shaped lipid bilayer membranous vesicular structures under a transmission electron microscope. The exosome diameter was approximately $30 \sim 150 \mathrm{~nm}$, and cell debris and suborganelles were not observed in the entire field of view (Fig. 1A). The nanoparticle analysis results indicated that the particle diameter of the exosomes was approximately $30 \sim 150$ $\mathrm{nm}$, and the distribution peak was $72 \mathrm{~nm}$ (Fig. 1B). Western blot analysis showed the obvious expression of the exosome protein markers CD9, Hsp70 and Alix, but not Golgi matrix protein 130(GM130) (Fig. 1C). Flow cytometry revealed the expression of CD63 and CD81, with a positive expression rate of more than $70 \%$ (Fig. 1D).
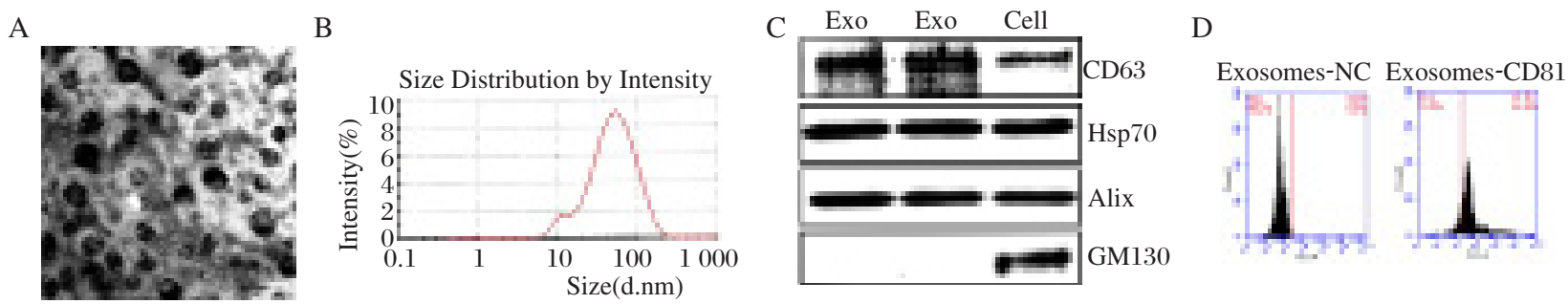

Fig1 The purification and identification of exosomes. A: Morphological observation by transmission electron microscopy; B: NanoSight nanoparticle analysis; C: Molecular phenotyping (Western blotting); D: Flow cytometry result.

\section{Expression of miR-375 in exosomes (RT-PCR)}

Sequence amplification primers were designed to detect the exosomal miR-375 in PCa cell lines (LNCaP,PC-3,22RV1 and DU-145) and normal prostate epithelial cells (RWPE-1) .The level of exosomal miR-375 isolated from PCa cells was significantly higher than the expression level of miR375 from normal RWPE-1 prostate epithelial cells $(P<0.05$,Fig.2A $)$. Subsequently, a high miR-375-expressing $\mathrm{PCa}$ cell line ( $\mathrm{LNCaP})$ and a low miR375-expressing normal prostate epithelial cell line (RWPE-1) were used to analyze the differences in exosomal miRNA expression by NGS.

\section{Analysis of differences in NGS expression}

The exosome sequencing libraries of LNCAP and RWPE-1 cells were sequenced and filtered by Illumina HiSeqTM2500. The obtained sequences were compared with those in the miRBase 21.0 database, and information was obtained for each component and its expression of content information. The average percentage of miRNA in the sample was $6.51 \%$ (Fig. 2B). The difference between the expression of miRNA in the groups was compared by scatterplot analysis using the Log2 ratio (Fig. 2C). Compared with the control group, the PCa cell group exhibited 298 differentially expressed genes (ILog2(foldchange) $\mid>1$ ), with 198 down-regulated and 100 upregulated miRNAs. Among these molecules, 50 miRNAs were significantly differentially expressed $(P<0.01)$, with 37 significantly down-regulated miRNAs and 13 significantly up-regulated miRNAs (Table 1). This study identified 50 differentially ex- pressed miRNAs, and cluster analysis was able to fully distinguish between the two types of exosomes (Fig. 2D). In exosomes from LNCaP, miR-375, miR200c-3p and miR-141-3p showed up-regulated expression, whereas miR-100-5p, miR-584-5p, and miR-125b-1-3p showed down-regulated expression (Fig. 2E).

\section{Osteoblast activity was promoted by exoso- mal miR-375}

The CMV-RFP-CD63 lentivirus was used to transfect LNCAP and RWPE-1 cells to generate exosomes labelled with red fluorescent protein (RFP+) and then construct a cell co-culture system. After a total of $72 \mathrm{~h}$, clearly visible red fluorescent protein (RFP+) was found in osteoblasts (OB) cultured with LNCaP cells (Fig.3A). The level of miR-375 was noticeably increased in osteoblasts cultured with LNCaP cells (Fig.3B). Additionally, the proliferative activity of osteoblasts was significantly increased after co-culture with LNCaP cells (Fig.3C). Next, by transfecting osteoblasts with miR-375 mimics, we explored whether the changes in the proliferation activity of osteoblasts were related to the level of exosomal miR-375.

After transfecting osteoblasts with miR-375 mimics, alkaline phosphatase (ALP) staining showed a large number of brown ALP particles in the cytoplasm. Additionally, a significant increase in ALP activity was observed relative to the control group $(P<0.01$,Fig. $4 \boldsymbol{A})$. The results of alizarin red staining revealed apparent orange-red calcium nodules in osteoblasts. Quantitative analysis revealed that the extent of extracellular matrix 
Table 1 Significant differences in miRNA expression among samples

\begin{tabular}{|c|c|c|c|c|c|}
\hline miRNA_ID & $\mathrm{LNCaP}$ & RWPE-1 & up/down & $\log 2(\mathrm{FC})$ & $P$-value \\
\hline hsa-miR-375 & 660.546 & 4.847 & up & -7.090 & $<0.001$ \\
\hline hsa-miR-200c-3p & 117.042 & 1.454 & up & -6.330 & $<0.001$ \\
\hline hsa-miR-100-5p & 28.453 & 93514.788 & down & 11.682 & $<0.001$ \\
\hline hsa-miR-221-3p & 0.370 & 438.183 & down & 10.207 & $<0.001$ \\
\hline hsa-miR-584-5p & 0.574 & 1336.401 & down & 11.184 & $<0.001$ \\
\hline hsa-miR-141-3p & 92.104 & 0 & up & -7.716 & $<0.001$ \\
\hline hsa-miR-383-5p & 6.437 & 0.133 & up & -5.589 & $<0.001$ \\
\hline hsa-miR-125b-1-3p & 0.113 & 737.556 & down & 12.663 & $<0.001$ \\
\hline hsa-miR-222-3p & 0.114 & 461.820 & down & 11.97 & $<0.001$ \\
\hline hsa-miR-1275 & 7.608 & 0.280 & up & -4.76 & $<0.001$ \\
\hline hsa-miR-3180 & 29.633 & 2.739 & up & -3.435 & $<0.001$ \\
\hline hsa-miR-4483 & 3.830 & 0.357 & up & -3.420 & $<0.001$ \\
\hline hsa-miR-148a-3p & 622.901 & 530.226 & up & -3.529 & $<0.001$ \\
\hline hsa-miR-4683 & 0.087 & 74.090 & down & 9.727 & $<0.001$ \\
\hline hsa-miR-92a-1-5p & 4.492 & 0.637 & up & -2.817 & $<0.001$ \\
\hline hsa-miR-128-1-5p & 4.455 & 0.609 & up & -2.869 & $<0.001$ \\
\hline hsa-miR-1273g-3p & 6.629 & 1.770 & up & -1.904 & $<0.001$ \\
\hline hsa-miR-146a-5p & 0.989 & 132.748 & down & 7.067 & $<0.001$ \\
\hline hsa-miR-424-3p & 0.591 & 107.159 & down & 7.502 & $<0.001$ \\
\hline hsa-miR-125b-5p & 6.303 & 531.265 & down & 6.397 & $<0.001$ \\
\hline hsa-miR-196a-5p & 26.85 & 3.374 & up & -2.992 & $<0.001$ \\
\hline hsa-miR-134-5p & 0.117 & 22.589 & down & 7.591 & $<0.001$ \\
\hline hsa-miR-3135b & 17.690 & 4.837 & up & -1.870 & $<0.001$ \\
\hline hsa-miR-873-3p & 0.655 & 33.700 & down & 5.685 & $<0.001$ \\
\hline hsa-miR-95-3p & 0.195 & 11.800 & down & 5.915 & $<0.001$ \\
\hline hsa-miR-210-3p & 0.532 & 58.437 & down & 6.77 & $<0.001$ \\
\hline hsa-miR-99b-5p & 100.091 & 3353.701 & down & 5.066 & $<0.001$ \\
\hline hsa-miR-4446-3p & 0.073 & 13.212 & down & 7.493 & $<0.001$ \\
\hline hsa-miR-9-5p & 4.995 & 336.572 & down & 6.074 & $<0.001$ \\
\hline hsa-miR-3934-5p & 1.121 & 37.106 & down & 5.048 & $<0.001$ \\
\hline hsa-miR-421 & 0.493 & 20.191 & down & 5.354 & $<0.001$ \\
\hline hsa-miR-139-5p & 0.425 & 20.522 & down & 5.590 & $<0.001$ \\
\hline hsa-miR-183-5p & 74.134 & 3554.843 & down & 5.583 & $<0.001$ \\
\hline hsa-miR-381-3p & 0.988 & 40.863 & down & 5.370 & $<0.001$ \\
\hline hsa-miR-24-3p & 109.163 & 3243.622 & down & 4.893 & $<0.001$ \\
\hline hsa-miR-92a-3p & 337.962 & 17202.180 & down & 5.669 & $<0.001$ \\
\hline hsa-miR-21-5p & 153.241 & 4900.051 & down & 4.998 & $<0.001$ \\
\hline hsa-miR-192-5p & 13.447 & 537.908 & down & 5.322 & 0.001 \\
\hline hsa-miR-126-3p & 1.723 & 68.249 & down & 5.307 & 0.001 \\
\hline hsa-let- $7 \mathrm{e}-5 \mathrm{p}$ & 3.712 & 83.898 & down & 4.498 & 0.002 \\
\hline hsa-let-7a-5p & 225.265 & 5351.823 & down & 4.570 & 0.003 \\
\hline hsa-miR-532-5p & 114.652 & 3253.283 & down & 4.826 & 0.003 \\
\hline hsa-miR-625-3p & 6.360 & 155.272 & down & 4.609 & 0.004 \\
\hline hsa-miR-125a-3p & 8.542 & 193.385 & down & 4.500 & 0.004 \\
\hline hsa-miR-409-3p & 4.524 & 109.785 & down & 4.601 & 0.005 \\
\hline hsa-miR-128-3p & 88.752 & 2177.163 & down & 4.616 & 0.006 \\
\hline hsa-miR-182-5p & 38.802 & 960.206 & down & 4.629 & 0.006 \\
\hline hsa-miR-101-3p & 32.976 & 1446.715 & down & 5.455 & 0.006 \\
\hline hsa-miR-548o-3p & 1.337 & 38.338 & down & 4.841 & 0.006 \\
\hline
\end{tabular}


A

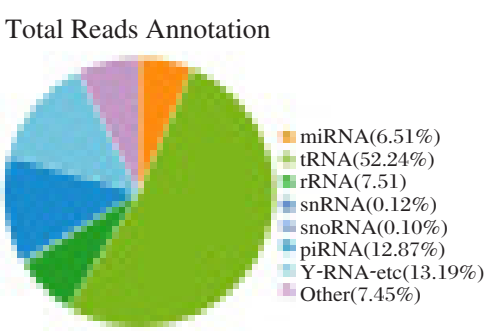

D

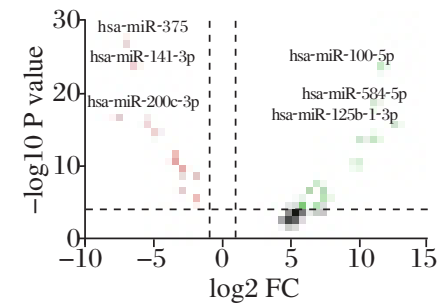

B
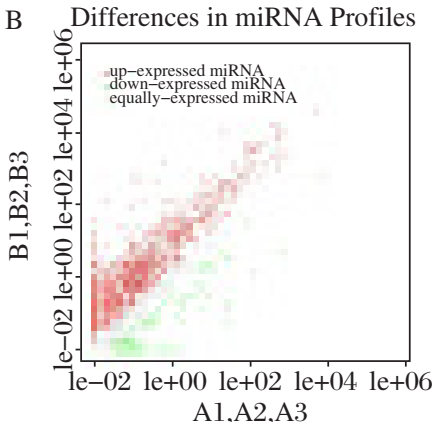

E

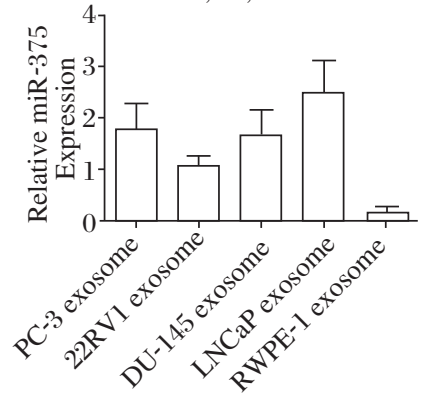

C
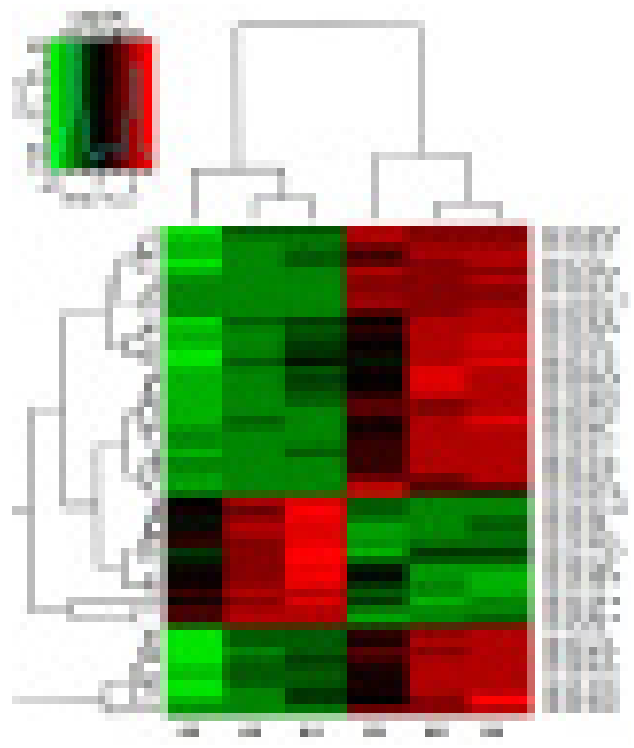

Fig 2 The differential expression of exosomal miR-375. A: Level of miR-375 in exosomes; B: Non-coding RNA classification and annotation(A1-3 group : LNCaP; B1-3 group: RWPE-1. E); C: Scatter plot of differences in miRNA expression; D: Heatmap of differences in miRNA expression; E: Volcano plot of differences in miRNA expression.

A
$\mathrm{OB}$
$(\mathrm{DAPI})$

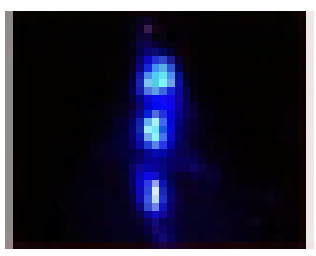

OB

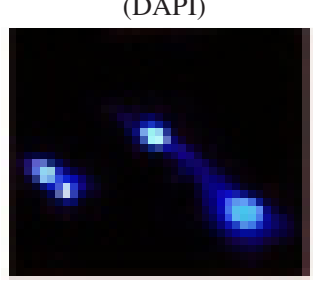

LNCaP-exo (CMV-RFP-CD63)

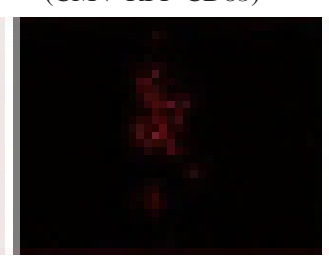

RWPE-1-exo

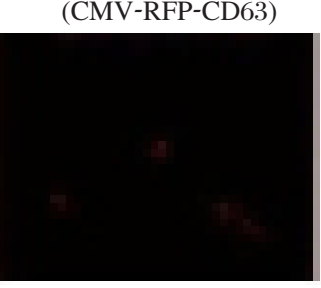

Merge

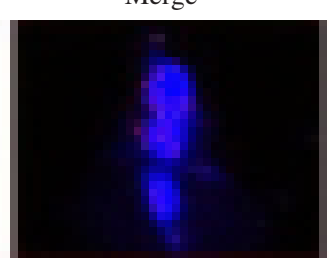

Merge

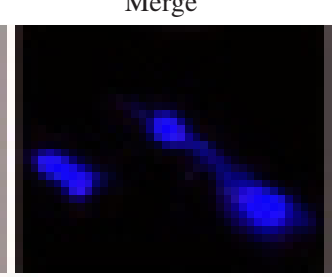

B

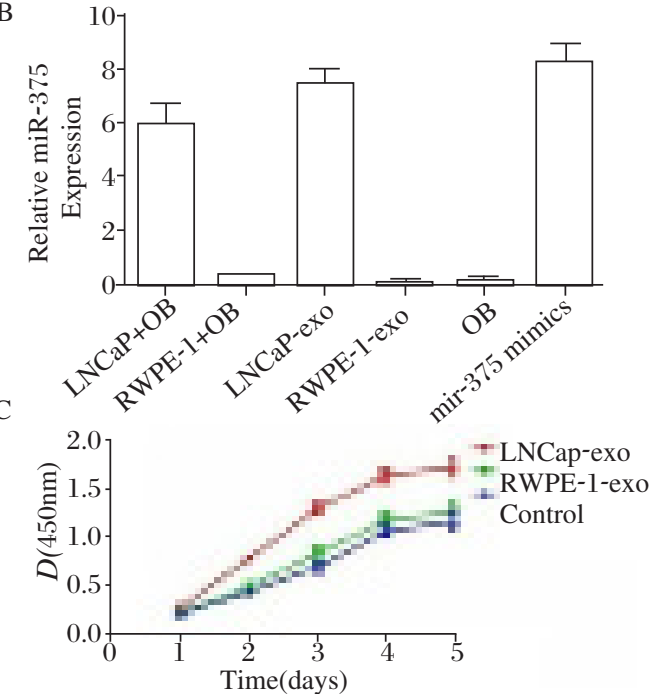

Fig 3. The effects of exosomes from LNCaP cells on osteoblasts. A: CD63-RFP-CD63 lentiviral tracer; B: Changes in miR-375 expression; $\mathrm{C}$ : Changes in the proliferative activity of osteoblasts.

mineralization in the group of osteoblasts transfected with miR-375 mimics was significantly greater than that in the control group $(P<0.05$, Fig.4B $)$. Furthermore, the proliferative activity of osteoblasts was significantly increased in the group transfected with miR-375 mimics (Fig.4C). RT-PCR results showed that osteoblasts transfected with miR-375 mimics overexpressed Opg, Opn, Runx2 and Bsp(Fig.4D).

\section{DISCUSSION}

In a previous study, Johnstone et al. ${ }^{[16]}$ isolated and extracted exosomes from reticulocyte culture medium, which harbours a type of membranous vesicle that is released into the extracellular matrix by the fusion of cellular multi-vesicle bodies and cytoplasmic membranes. Studies have shown that various cells, including tumour cells, can form and secrete exosomes. Exosomes from different cells carry the proteins, lipids and nucleic acids of their parental cells, and participate in intercellular communication to regulate the tumour metastasis microenvironment $^{[17]}$. In this study, we used morphological observation and particle size analysis to confirm that the cellular extract contained morphological characteristics of exosomes.

Exosomes contain a variety of proteins, DNA, mRNA, miRNA and long-chain non-coding RNA ${ }^{[18]}$. Almost all exosomes harbour a common protein 


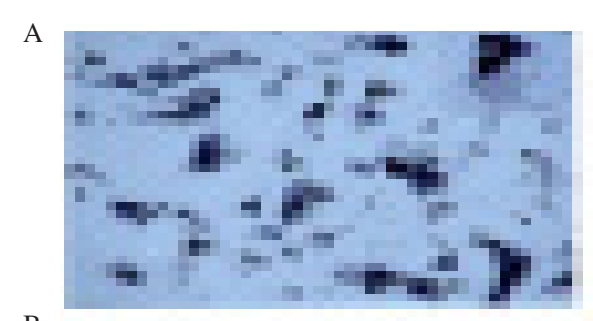

B

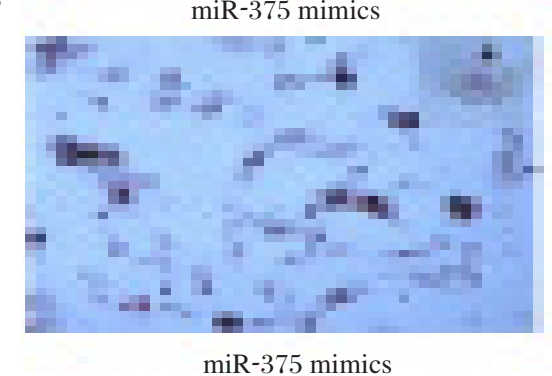

$\mathrm{C}$

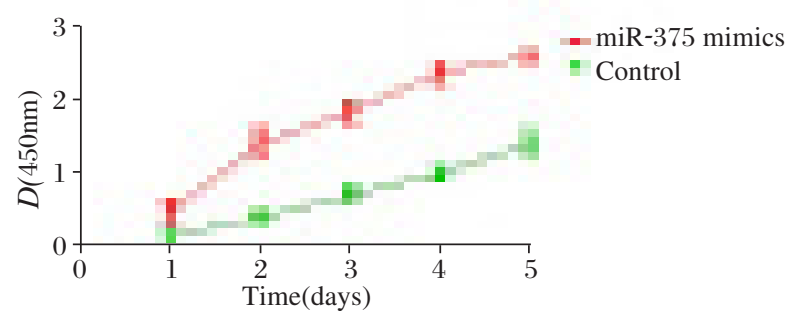

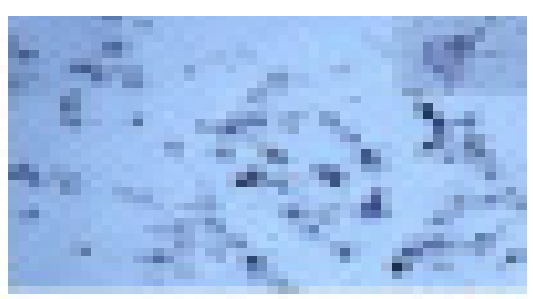

Control

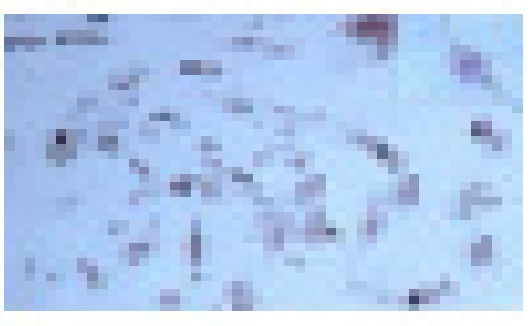

Control

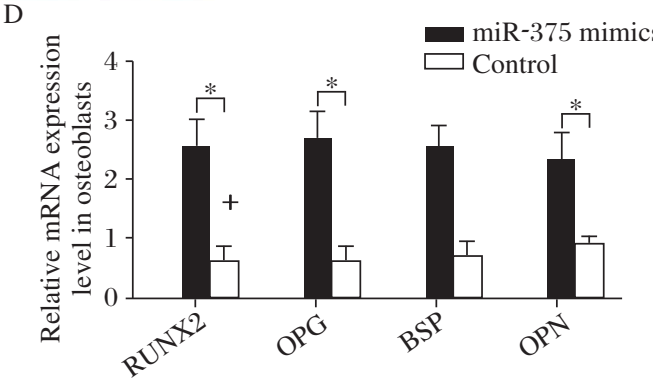

Fig 4. The roles of miR-375 mimics on osteoblast activity. A: Alkaline phosphatase staining and alkaline phosphatase activity; B: Alizarin red staining and the level of extracellular matrix mineralization; C: Cell proliferative activity; D: Expression of osteoblast activity-related marker genes, ${ }^{*} P<0.05$.

or protein family that can be used as an exosome marker. These proteins include membrane-associated proteins CD9, CD63, CD81 and CD82; cytoplasmic proteins Hsp70 and Hsp90; important components of the internal body separation complex, including Alix and TSG101, membrane transport and fusion proteins Rab and GTP, and membrane proteins such as Annexins ${ }^{[19,20]}$. In this study, five protein markers (CD9, CD63, CD81, Hsp70 and Alix) were selected as positive indicators of exosomes, while GM130 was used as a negative marker. The extract isolated from the cell culture medium was consistent with molecular phenotypic characteristics of exosomes through identification of specific markers.

Studies have demonstrated altered levels of miRNAs in the development of $\mathrm{PCa}$, with differences between PCa patients and healthy individuals ${ }^{[21,22]}$. Our results showed that the expression level of exosomal miR375 was significantly increased in $\mathrm{PCa}$ cells $(P<0.05)$ and that exosomes from $\mathrm{LNCaP}$ cells demonstrated increased expression of this miRNA. Compared with RWPE-1 cells, the top three miRNA molecules with up-regulated expression were miR-375, miR-200c$3 p$ and miR-14l-3p, while the top three miRNA molecules with down-regulated expression were miR-100-5p, miR-584-5p, and miR-125b-1-3p in exosomes from LNCaP cells. Cancer cells are reported to secrete a large number of exosomes, which are rich in miRNAs that can integrate into target cells and affect their physiological function ${ }^{[23]}$. Most importantly, these results suggest that exosomemediated miRNA transport plays an important role in cancer development. There are many reports about the relationship between miRNA and PCa research, involving miRNAs such as miR-375, 21, 141, the let $-7 \mathrm{a}$, etc $^{[22,24]}$. In this study, the results of the NGS differential expression analysis further confirmed the high expression of miR-375 in the exosomes of LNCaP cells.

Bone metastasis is the most common complication in the advanced stages of PCa. This disease is characterized by bone lesions, and the truncal bones is a common site. Bone metastasis is also the leading cause of death in patients with $\mathrm{PCa}^{[25]}$. In recent years, studies on bone metastasis in PCa have received significant attention. Several studies support Stephen Paget's theory of "seed soil", suggesting that invasive tumour cells can only proliferate in microenvironments suitable for their growth and that they form metastatic lesions in specific tissues and organs ${ }^{[26]}$. Hoshino et al ${ }^{[27]}$ indicated that exosomes can enter the blood circulation and reach metastatic loci 
before cancer cells, thereby stimulating the microenvironment and promoting metastasis. Silva found that exosomal MIF primes the liver for metastasis and may be a prognostic marker for the development of PDAC liver metastasis ${ }^{[14]}$. A research team at Harvard University revealed the impact of the microenvironment on tumour growth and metastasis, showing that tumour cells and osteoblasts can remotely interact with and promote the proliferation of one another ${ }^{[28]}$. Increasing evidence suggests that the development of PCa is closely related to the interaction of tumour cells and the microenvironment ${ }^{[29]}$. Osteoblasts are an important type of cell in the bone metastasis microenvironment of $\mathrm{PCa}$ and are involved in the synthesis of bone matrix and mineralization, bone growth and development, damage and repair, and important regulatory functions. The differentiation and maturation of osteoblasts involve many factors, among which miRNAs play an important role ${ }^{[30]}$.

In this study, a large number of $\mathrm{RFP}^{+}$particles were found in osteoblasts cultured with LNCaP cells, the level of miR-375 in osteoblasts was significantly increased, and cell proliferation activity was significantly enhanced. ALP is an extracellular enzyme secreted by osteoblasts, and it can be used as an important index for the early differentiation of osteoblasts ${ }^{[31]}$. The results of our ALP staining showed a large number of brown alkaline phosphatase particles in the cytoplasm of osteoblasts after transfection. The detection of ALP activity was significantly increased compared with that in the control group $(P<0.01)$. Calcium nodules are important markers in the late stages of osteoblast differentiation, indicating the level of extracellular matrix mineralization. Alizarin red staining is a common method to observe the formation of calcium nodules and determine the degree of osteoblast differentiation, as calcium ions in calcium nodules can be chelated with alizarin red to form a red complex ${ }^{[32]}$. In this study, the results of alizarin red staining revealed the obvious deposition of orange-red calcium nodules in osteoblasts after transfection. Quantitative analysis showed that the level of extracellular matrix mineralization by osteoblasts after transfection was significantly higher than that in the control group $(P<0.05)$. Furthermore, compared with control cells, the miR-375 mimictransfected osteoblasts had significantly increased expression of Opg, Runx2, Opn and Bsp, which are related to osteoblast activity and differentiation.

In conclusion, our findings suggest that miR-375 is highly expressed in exosomes from the PCa cell line LNCaP and that exosomes can enter osteoblasts in large numbers and increase the level of miR-375. In addition, exosomal miR-375 can significantly promote osteoblast activity. Thus, the present study lays the foundation for further studies on the role of exosomal miR-375 in the activation and differentiation of osteoblasts and the mechanism of bone metastasis in PCa.

\section{Acknowledgements and funding}

The authors would like to thank the study subjects and research personnel for their involvement in the study. The authors would also like to thank Professor Jiang Zhang and Professor Chen Shao for their excellent theoretical assistance. This study was supported by the fund of the First Affiliated Hospital of Xi'an Medical University (XYFY14-02).

\section{References}

[1] Ferlay J, Shin HR, Bray F, et al. Estimates of worldwide burden of cancer in 2008: GLOBOCAN 2008[J]. Int J Cancer, 2010,127(12):2893-2917

[2] Siegel RL, Miller KD, Jemal A. Cancer statistics, 2017[J]. CA Cancer J Clin, 2017,67(1):7-30

[3] Suzman DL, Boikos SA, Carducci M. Bone-targeting agents in prostate cancer[J]. Cancer Metastasis Rev, 2014,33(2/3):619-628

[4] Larson SR, Zhang X, Dumpit R, et al. Characterization of osteoblastic and osteolytic proteins in prostate cancer bone metastases[J]. Prostate, 2013,73(9):932-940

[5] Rajpar S, Fizazi K. Bone targeted therapies in metastatic castration-resistant prostate cancer[J]. Cancer J, 2013,19(1):66-70

[6] Johnstone RM, Adam M, Hammond JR, et al. Association of plasma membrane activities with released vesicles (exosomes)[J]. Biol Chem, 1987, 262(19):94129420.

[7] Denzer K, Kleijmeer MJ, Heijnen HF, et al. Exosome: from internal vesicle of the multivesicular body to intercellular signaling device[J]. J Cell Sci, 2000,113(Pt 19):3365-3374

[8] Gusachenko ON, Zenkova MA, Vlassov VV. Nucleic acids in exosomes: disease markers and intercellular communication molecules[J]. Biochemistry (Mosc), 2013,78(1):1-7

[9] Soung YH, Ford S, Zhang V, et al. Exosomes in cancer diagnostics[J]. Cancers(Basel), 2017,9(1): pii: E8. doi: 10.3390/cancers9010008.

[10] Ruivo CF, Adem B, Silva M, et al. The biology of cancer exosomes: insights and new perspectives[J]. Cancer Res, 2017,77(23):6480-6488

[11] Zhou M, Chen J, Zhou L, et al. Pancreatic cancer derived exosomes regulate the expression of TLR4 in dendritic cells via miR-203[J]. Cell Immunol, 2014,292(1/2):65-69

[12] Stevanato L, Thanabalasundaram L, Vysokov N, et al. Investigation of content, stoichiometry and transfer 
of miRNA from human neural stem cell line derived exosomes[J]. PLoS One, 2016,11(1):e0146353

[13] Greening DW, Gopal SK, Xu R, et al. Exosomes and their roles in immune regulation and cancer[J]. Semin Cell Dev Biol, 2015,40:72-81

[14] Costa-Silva B, Aiello NM, Ocean AJ, et al. Pancreatic cancer exosomes initiate premetastatic niche formation in the liver[J]. Nat Cell Biol, 2015,17(6):816-826

[15] Möller A,House CM,Wong CS,et al. Inhibition of Siah ubiquitin ligase function[J]. Oncogene, 2009,28(2):289296

[16] Johnstone RM, Adam M, Hammond JR, et al. Vesicle formation during reticulocyte maturation $[\mathrm{J}]$. Association of plasma membrane activities with released vesicles(exosomes)[J]. Biol Chem,1987,262(19):94129420

[17] Ge R, Tan E, Sharghi-Namini S, et al. Exosomes in cancer microenvironment and beyond: have we overlooked these extracellular messengers?[J]. Cancer Microenviron, 2012,5(3):323-332

[18] Yu S, Cao H, Shen B, et al. Tumor-derived exosomes in cancer progression and treatment failure $[\mathrm{J}]$. Oncotarget, 2015,6(35):37151-37168

[19] Mathivanan S, Fahner CJ, Reid GE, et al. ExoCarta 2012: database of exosomal proteins, RNA and lipids[J]. Nucleic Acids Res, 2012,40(D1):D1241-D1244

[20] Théry C, Ostrowski M, Segura E. Membrane vesicles as conveyors of immune responses[J]. Nat Rev Immunol, 2009,9(8):581-593

[21] Bertoli G, Cava C, Castiglioni I. MicroRNAs as biomarkers for diagnosis, prognosis and theranostics in prostate cancer[J]. Int J Mol Sci, 2016,17(3):421

[22] Filella X, Foj L. miRNAs as novel biomarkers in the management of prostate cancer[J]. Clin Chem Lab Med, 2017,55(5):715-736
[23] Brinton LT, Sloane HS, Kester M, et al. Formation and role of exosomes in cancer[J]. Cell Mol Life Sci, 2015,72:659-671.

[24] Porzycki P, Ciszkowicz E, Semik M, et al. Combination of three miRNA(miR-141, miR-21, and miR-375) as potential diagnostic tool for prostate cancer recognition[J]. Int Urol Nephrol, 2018,50(9):1619-1626

[25] Rucci N, Angelucci A. Prostate cancer and bone: the elective affinities[J]. Biomed Res Int. 2014,2014: 167035.

[26] Paget $\mathrm{S}$. The distribution of secondary growths in cancer of the breast. 1889[J]. Cancer Metastasis Rev, 1989,8(2):98101

[27] Hoshino A, Costa-Silva B, Shen TL, et al. Tumour exosome integrins determine organotropic metastasis[J]. Nature,2015,527(7578):329-335

[28] Engblom C, Pfirschke C, Zilionis R, et al. Osteoblasts remotely supply lung tumors with cancerpromoting SiglecFhigh neutrophils[J]. Science, 2017,358(6367):5801

[29] San Martin R, Pathak R, Jain A, et al. Tenascin-C and integrin $\alpha 9$ mediate interactions of prostate cancer with the bone microenvironment[J]. Cancer Res, 2017,77(21):5977-5988

[30] Papaioannou G, Mirzamohammadi F, Kobayashi T. MicroRNAs involved in bone formation[J]. Life Sci, 2014,71(24):4747-4761

[31] Yun HM, Park KR, Quang TH, et al. 2,4,5-Trimethoxyldalbergiquinol promotes osteoblastic differentiation and mineralization via the BMP and $\mathrm{Wnt} / \beta$-catenin pathway[J]. Cell Death Dis, 2015,6(7):e1819

[32] Lee HS, Jung EY, Bae SH, et al. Stimulation of osteoblastic differentiation and mineralization in MC3T3-E1 cells by yeast hydrolysate[J]. Phytother Res, 2011,25(5):716-723

(Received 19 September 2018, Revised 08 October 2018, Accepted 25 October 2018) 